\title{
Explicit Schoen surfaces
}

\author{
Carlos Rito, Xavier Roulleau and Alessandra Sarti
}

\begin{abstract}
We give an explicit construction for the 4-dimensional family of Schoen surfaces by computing equations for their canonical images, which are 40-nodal complete intersections of a quadric and the Igusa quartic in $\mathbb{P}^{4}$. We then study a particularly interesting example, with 240 automorphisms and maximal Picard number.
\end{abstract}

\section{Introduction}

While working on a problem related to the Hodge conjecture, Schoen [Sch07] used deformation theory to construct a family of surfaces $S$ (from now on called Schoen surfaces) with some interesting features. The one that has interested Schoen most is that the Albanese map of $S$ embeds it into its Albanese variety $A$, but for a generic Schoen surface, the cycle $S$ in $H^{4}(A, \mathbb{Q})$ is not contained in the subspace generated by the intersections of two divisors of $A$; the existence of such cycles on an abelian variety $A$ makes the Hodge conjecture more difficult to prove.

Another interesting property of $S$ is that the natural map

$$
\wedge^{2} H^{0}\left(S, \Omega_{S}\right) \rightarrow H^{0}\left(S, K_{S}\right)
$$

has a 1-dimensional kernel, and therefore $S$ is a Lagrangian surface in $A$. Moreover, the kernel is not of the form $\omega_{1} \wedge \omega_{2}$, with $\omega_{i} \in H^{0}\left(S, \Omega_{S}\right)$; therefore, by the Castelnuovo-De Franchis theorem, the surface does not admit fibrations onto a curve of genus at least 2. Only a few Lagrangian surfaces without a fibration onto a curve of higher genus are known (see [BNP07, BPS10, BT00]). Such examples are interesting for people studying Kählerian groups; for example, one can ask whether their fundamental group is nilpotent (cf. [Cam95]).

By [Bea79], when the canonical map of a surface of general type has degree at least 2 onto a surface, that surface either has $p_{g}=0$ or is itself canonically embedded, the latter case being rather exceptional (see [CPT03] for a list of the examples known so far). In [CMR15], Ciliberto, Mendes Lopes and the second author studied Schoen surfaces geometrically, proving that the canonical map of a Schoen surface $S$ is 2-to-1 onto a 40-nodal, degree 8, complete intersection surface $X_{40} \subset \mathbb{P}^{4}$ and the ramification of the double cover $S \rightarrow X_{40}$ is the set of 40 nodes. They also show that Schoen surfaces are not universally covered by the bidisk (very few surfaces with $K^{2}=8 \chi$ and this property are known).

Received 1 January 2017, accepted in final form 5 March 2018.

2010 Mathematics Subject Classification 14J29, 14J28.

Keywords: irregular surfaces, Lagrangian surfaces, Igusa quartic, Segre cubic, K3 surfaces.

This journal is (C) Foundation Compositio Mathematica 2019. This article is distributed with Open Access under the terms of the Creative Commons Attribution Non-Commercial License, which permits non-commercial reuse, distribution, and reproduction in any medium, provided that the original work is properly cited. For commercial re-use, please contact the Foundation Compositio Mathematica.

This research was partially supported by FCT (Portugal) under the project PTDC/MAT-GEO/2823/2014, the fellowship SFRH/BPD/111131/2015 and by CMUP (UID/MAT/00144/2019), which is funded by FCT with national (MEC) and European structural funds through the programs FEDER, under the partnership agreement PT2020. 


\section{EXPLICIT SCHOEN SURFACES}

Miyaoka's bound tells us that on a degree 8 complete intersection surface in $\mathbb{P}^{4}$, there cannot be more than 40 nodes. The construction of Schoen surfaces gives the first theoretical proof that such a 40-nodal surface exists, but without providing any equations for it. In [Bea13], Beauville used Schoen surfaces in order to show the existence of 48-nodal degree 16 complete intersection surfaces $X_{48}$ in $\mathbb{P}^{6}$ and surfaces $\tilde{S}$ whose canonical map is 2-to-1 onto $X_{48}$.

The main result of this paper is an explicit construction of the surfaces $X_{40}$ by equations, and therefore an explicit construction of $S$ by double cover. The idea of the construction of the surfaces $X_{40}$ is the following. The Igusa quartic threefold $I_{4} \subset \mathbb{P}^{4}$ is singular along 15 lines of $A_{1}$-singularities. Its intersection with a generic quadric thus gives a degree 8 complete intersection surface containing 30 nodes. The main question is therefore to find a quadric $Q_{2}$ which, while still transversal to the 15 singular lines, is tangent to the Igusa quartic at 10 more points, leading to a 40-nodal surface $X_{40}:=I_{4} \cap Q_{2}$. Our construction is very concrete since we have the explicit equation for the quadric $Q_{2}$, whose coefficients depend on four parameters.

In order to obtain that result, we use the knowledge of the above mentioned papers, computer algebra and the rich geometry of the Igusa quartic threefold and of its dual, the Segre cubic threefold $S_{3} \subset \mathbb{P}^{4}$, which is the unique cubic threefold with 10 nodes. It is well known that the Igusa quartic threefold parametrizes quartic Kummer surfaces [Hun96, Theorem 3.3.8]: taking the intersection of $I_{4}$ by a hyperplane $T_{x}$ tangent to a generic point $x \in I_{4}$, one obtains a 16nodal Kummer surface, 15 nodes coming from the intersection of the 15 lines in $I_{4}$ with $T_{x}$, and one more node at $x$. Our construction of $X$ uses the 15-nodal $K 3$ surfaces obtained as the intersections of $I_{4}$ with a generic hyperplane, giving new interesting geometric features to the Igusa quartic $I_{4}$.

We then study a Schoen surface with a large group of symmetries (of order 240). We compute the isogeny class of its Albanese variety, and we prove that it has maximal Picard number. Although interesting, examples of surfaces with maximal Picard number are rather scarce; see, for example, [Bea14].

As a by-product of our work, we also obtain a geometric construction of a 3-dimensional subfamily of Schoen surfaces, as a bidouble cover of some particular Kummer surfaces. This construction interestingly matches some (theoretical) constructions of Lagrangian surfaces suggested by Bogomolov and Tschinkel in [BT00] (see Remark 13).

Since 15-nodal quartic surfaces, obtained as generic hyperplane sections of the Igusa quartic, play a key role in our construction, one may ask if an analogous construction could be done using a different family of 15-nodal quartics. The answer is negative: we show in the appendix that a generic quartic surface with 15 nodes can be realized as a hyperplane section of the Igusa quartic threefold.

The paper is organized as follows. In Section 2, we recall some known facts on Schoen surfaces. In Section 3, we construct the 40-nodal degree 8 surfaces in $\mathbb{P}^{4}$, we prove that their sets of 40 nodes are 2-divisible, their associated double covers are not universally covered by the bidisk and that they are Schoen surfaces. In Section 4, we study an example of a Schoen surface with a large automorphism group. The appendix is on the moduli of K3 surfaces with 15 nodes.

Notation. We work over the complex numbers. All varieties are assumed to be projective algebraic. For a smooth surface $S$, as usual, $K_{S}$ is the canonical class, $p_{g}(S):=h^{0}\left(S, K_{S}\right)$ is the geometric genus, $q(S):=h^{1}\left(S, K_{S}\right)$ is the irregularity, and $\chi\left(\mathcal{O}_{S}\right)=1-q+p_{g}$ is the holomorphic 


\section{Rito, X. Roulleau and A. Sarti}

Euler characteristic. A $(-n)$-curve on a surface is a curve isomorphic to $\mathbb{P}^{1}$ with self-intersection $-n$. Linear equivalence of divisors is denoted by $\equiv$.

\section{Schoen surfaces}

Let $C$ be a smooth genus 2 curve with Jacobian $J(C)$, and consider the union

$$
V:=C \times C \cup_{C} J(C)
$$

glued along the diagonal of $C \times C$ and $C \hookrightarrow J(C)$. Notice that $V$ is singular along $C$.

ThEOREM 1 ([Sch07]). The reducible surface $V$ can be deformed into a smooth surface of general type $S$ with invariants

$$
c_{1}^{2}=16=2 c_{2}, \quad q=4, \quad p_{g}=5 .
$$

The moduli space of these surfaces is 4-dimensional. The deformation space is locally smooth; thus, it is locally irreducible.

As said in the introduction, the canonical map of a Schoen surface $S$ is of degree 2 onto a 40-nodal complete intersection $X$ of a quadric and a quartic in $\mathbb{P}^{4}$. From [CMR15, Lemmas 4.5 and 4.6 and proof of Theorem 4.1], we deduce the following.

Proposition 2 ([CMR15]). The above surfaces $X$ degenerate to the union of a double quadric surface and a quartic Kummer surface, glued along a trope of the Kummer surface. These surfaces are given by the intersection of two hyperplanes and a quartic hypersurface in $\mathbb{P}^{4}$. Moreover, this degeneration induces the degeneration in Schoen's construction.

\section{The construction}

In this section, we show the following.

Theorem 3. Let $I_{4}$ be the Igusa quartic in $\mathbb{P}^{4}$. There exists a quadric on four parameters $Q_{a, b, c, d}$ such that, for generic values of the parameters, the surface

$$
X_{40}:=I_{4} \cap Q_{a, b, c, d}
$$

has exactly 40 nodes. These nodes are 2-divisible in the Picard group, and the double cover $S \rightarrow X_{40}$ ramified over the nodes is a Schoen surface.

We explain how to compute the quadric $Q_{a, b, c, d}$. The corresponding computer code, implemented with Magma, is available at [Rit17].

\subsection{Segre cubic, Igusa quartic}

The linear system $L$ of quadrics through points $p_{1}, \ldots, p_{5} \in \mathbb{P}^{3}$ in general position (that is, no four of them are contained in a hyperplane) is 4 -dimensional. Let $\phi: \mathbb{P}^{3} \rightarrow \rightarrow \mathbb{P}^{4}$ be the rational map corresponding to the linear system $L$ on $\mathbb{P}^{3}$. The image $S_{3}:=\phi\left(\mathbb{P}^{3}\right)$ is the Segre cubic, the unique cubic threefold in $\mathbb{P}^{4}$ (up to projective equivalence) with singular set the union of 10 nodes (the images of the lines $p_{i} p_{j}$ ). The Segre cubic contains 15 planes: the "images" (after blowing up $\mathbb{P}^{3}$ ) of $p_{1}, \ldots, p_{5}$ and of the 10 planes in $\mathbb{P}^{3}$ through exactly three of the points $p_{i}$. The dual variety $I_{4}$ (the image under the gradient map) of $S_{3}$ is the Igusa quartic. The dual map contracts the above 15 planes to singular lines of $I_{4}$, its singular set. The Igusa quartic has 


\section{EXPLICIT SCHOEN SURFACES}

10 tropes, that is, 10 hyperplane sections which are double quadrics. For more details, see, for example, [Hun96] or [Dol12].

\subsection{The 40-nodal surface}

Let $H \subset \mathbb{P}^{4}$ be a generic hyperplane. Then $Q_{15}:=I_{4} \cap H \subset \mathbb{P}^{3}$ is a quartic surface with 15 nodes. Since $H$ is generic, we can choose five nodes $p_{1}, \ldots, p_{5}$ in general position (that is, no four of them are in a hyperplane). Consider the map $\phi: H \rightarrow \mathbb{P}^{4}$ given by the linear system $|L|$ of quadrics which pass through $p_{1}, \ldots, p_{5}$.

Proposition 4. There exists a quadric $S_{2} \subset \mathbb{P}^{4}$ such that

$$
Q_{10}:=\phi\left(Q_{15}\right) \cong S_{3} \cap S_{2}
$$

and $Q_{10}$ has 10 nodes which are disjoint from the nodes of $S_{3}$.

Proof. We have $\phi(H) \cong S_{3}$, and $\phi$ is of degree 1 outside of the lines $p_{i} p_{j}$; therefore, $\phi$ sends $Q_{15}$ birationally to a surface $Q_{10}$ contained in $S_{3}$, a 10-nodal $K 3$ surface. Now consider the resolution of singularities $\widetilde{Q}_{15} \rightarrow Q_{15}$, and let $\left|L^{\prime}\right|$ be the strict transform of $|L|$ in $\widetilde{Q}_{15}$. Since $L^{\prime 2}=6$ and $\left|L^{\prime}\right|$ has no base points, [Sai74, Theorem 6.1] implies that $Q_{10}$ is a complete intersection of a quadric $S_{2}$ and a cubic in $\mathbb{P}^{4}$. This cubic can be assumed to be $S_{3}$ because $Q_{10} \subset S_{3}$.

The second assertion follows from the fact that the 10 nodes of $Q_{10}$ correspond to the nodes of $Q_{15}$ disjoint from the 10 lines $p_{i} p_{j}$ for $i, j \in\{1, \ldots, 5\}$, which are contracted to the nodes of $S_{3}$.

Using Magma, we compute this 4-dimensional family of smooth quadrics $S_{2}$ (see [Rit17, Section A]). The dual of $S_{2}$ is a smooth quadric $Q_{2}$. Consider the dual maps

$$
\begin{aligned}
& d_{1}: S_{3} \rightarrow I_{4}, \\
& d_{2}: S_{2} \rightarrow Q_{2},
\end{aligned}
$$

and define $X_{40}:=I_{4} \cap Q_{2}$. Since $S_{2}$ is tangent to $S_{3}$ at 10 smooth points of $S_{3}$ and duality preserves tangencies, $X_{40}$ has at least 10 singular points. The purpose of this construction is to find $Q_{2}$ meeting the 15 singular lines of $I_{4}$ transversally, so that $X_{40}$ is a 40 -nodal surface. We show below that, up to the symmetry of $I_{4}$, at most one choice of the nodes $p_{1}, \ldots, p_{5}$ serves our aims. Notice that the quartic surface $Q_{15}$ has 10 tropes (double conics), which are induced by the tropes of $I_{4}$.

Proposition 5. If exactly three of the nodes $p_{1}, \ldots, p_{5}$ are in a trope of $Q_{15}$, then the surface $X_{40}$ is non-normal.

Proof. Let $T$ be the hyperplane of $H$ which gives the trope of $Q_{15}$ containing 3 of the nodes $p_{1}, \ldots, p_{5}$. Since $\phi$ is injective outside the union of the lines $p_{i} p_{j}$, one has $\phi\left(T \cap Q_{15}\right)=\phi(T) \cap S_{2}$. Let $C$ be the conic such that $T \cap Q_{15}=2 C$. When we blow up $\mathbb{P}^{3}$ at $p_{1}, \ldots, p_{5}$, the strict transform of a quadric in $|L|$ meets the strict transform of $C$ at exactly one point. This implies that the image $\phi(C)$ is a line. Then $\phi(T) \cap S_{2}$ is a double line and $\phi(T)$ is a plane in $S_{3}$, as stated in Section 3.1. When taking the duals, this plane is contracted to a singular line of $I_{4}$ (see, for example, [Hun96, Section 3.3.4]) and the quadric $Q_{2}$ contains this line. This implies that the singular set of $X_{40}$ is of dimension 1.

Therefore, a surface $X_{40}$ is normal only if no three of the nodes $p_{1}, \ldots, p_{5}$ are in a trope of $Q_{15}$. We compute that there are exactly six such sets of nodes (see [Rit17]). The 10 tropes 


\section{Rito, X. Roulleau and A. Sarti}

of $I_{4}$ induce 10 tropes of $Q_{15}$, so we compute the sets of five singular lines of $I_{4}$ such that there is no trope containing three of them. Fixing one of these sets (the $S_{6}$ symmetry of $I_{4}$ gives the remaining sets), we have a choice of five nodes $p_{1}, \ldots, p_{5}$ for each surface $Q_{15}$. The computations with Magma available in [Rit17] confirm that a generic surface $X_{40}$ constructed as above has 40 nodes and no other singularities. Our computations are optimal in the sense that we construct the entire family at once: the output is a quadric on four parameters $Q_{a, b, c, d}$ such that, for generic values of the parameters, the surface $I_{4} \cap Q_{a, b, c, d}$ has exactly 40 nodes.

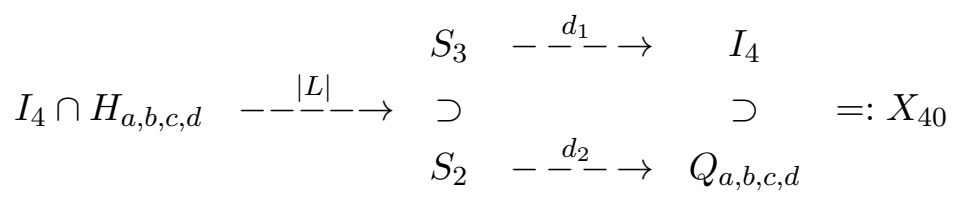

FIgURE 1. Construction of $X_{40}$, where the symbol $\supset$ means intersection

\subsection{2-divisibility of the nodes}

In the previous section, we proved that with a generic point $t$ in $\left(\mathbb{P}^{4}\right)^{*}$ (the dual space of $\mathbb{P}^{4}$ ), one can associate a surface $X_{t}$, the minimal resolution of a 40-nodal surface $X_{40}(t)$ in $\mathbb{P}^{4}$. Let $o$ be a point in $\left(\mathbb{P}^{4}\right)^{*}$ such that the surface $X_{o}$ is defined. There exist a small polydisk $B$ centered at $o$, a smooth complex manifold $\mathcal{X}$ and a proper flat morphism $\mathcal{X} \rightarrow B$ such that the fiber at $t \in B$ is $X_{t}$. There are moreover 40 divisors $D_{1}, \ldots, D_{40}$ on $\mathcal{X}$ such that the intersections of these divisors with $X_{t}$ are the forty $(-2)$-curves of $X_{t}$ over nodes in $X_{40}(t)$.

Proposition 6. Suppose that the sum of the forty $(-2)$-curves on $X_{o}$ is 2-divisible. Then, up to shrinking $B$, the sum of the forty (-2)-curves on the surface $X_{t}(t \in B)$ is 2-divisible.

Proof. Let $\mathcal{L}=\mathcal{O}_{\mathcal{X}}\left(\sum D_{i}\right)$. By hypothesis, there exists a line bundle $\mathcal{M}_{o}$ on $X_{o}$ such that $\mathcal{L}_{\mid X_{o}}=\mathcal{M}_{o}^{\otimes 2}$. The results follows from [CMR15, Lemma 2.2].

According to our computations (see [Rit17, Section B]), one of the 40-nodal surfaces constructed above is projectively equivalent to the $\Sigma_{5}$-invariant surface $\bar{X}_{40}$ given in $\mathbb{P}^{5}$ by

$$
\begin{gathered}
x+y+z+w+t+h=0, \\
5\left(x^{2}+\cdots+t^{2}\right)-7(x+\cdots+t)^{2}=0, \\
4\left(x^{4}+\cdots+t^{4}+h^{4}\right)-\left(x^{2}+\cdots+t^{2}+h^{2}\right)^{2}=0 .
\end{gathered}
$$

Proposition 7. The nodes of $\bar{X}_{40}$ are 2-divisible.

Proof. One can verify that $\bar{X}_{40}$ has a $(40,12)$ configuration: 40 tropes and 40 nodes, each trope contains 12 nodes, through each node pass 12 tropes. Using Magma (see [Rit17, Section C]), we show the existence of tropes $T_{1}, \ldots, T_{4}$ such that

- we have $T_{i}=2 C_{i}$, with $C_{2}, C_{3}, C_{4}$ smooth and $C_{1}$ the union of two conics;

- the singular points of $C_{1}$ are not in $C_{2} \cup C_{3} \cup C_{4}$;

- $C_{1} \cup C_{2}$ contains exactly 20 nodes of $\bar{X}_{40}$ which are not in $C_{1} \cap C_{2}$;

- $C_{3} \cup C_{4}$ contains exactly 20 nodes of $\bar{X}_{40}$ which are not in $C_{3} \cap C_{4}$;

- the above two sets of 20 nodes are disjoint. 


\section{EXPLICIT SCHOEN SURFACES}

Let $\widehat{X}_{40}$ be the smooth minimal model of $\bar{X}_{40}$. Denote by $\widetilde{T}_{i}$ the total transform of $T_{i}$ in $\widehat{X}_{40}$ and by $\widehat{C}_{i}$ the strict transform of $C_{i}$ in $\widehat{X}_{40}$, for $i=1, \ldots, 4$. There are $(-2)$-curves $A_{1}, \ldots, A_{22} \subset$ $\widehat{X}_{40}$ such that

$$
\begin{aligned}
& \widetilde{T}_{1} 2 \widehat{C}_{1}+\sum_{1}^{10} n_{i} A_{i}+n_{21} A_{21}+n_{22} A_{22}, \\
& \widetilde{T}_{2} 2 \widehat{C}_{2}+\sum_{11}^{20} n_{i} A_{i}+n_{21}^{\prime} A_{21}+n_{22}^{\prime} A_{22}
\end{aligned}
$$

for some integers $n_{1}, \ldots, n_{22}, n_{21}^{\prime}, n_{22}^{\prime}$. From $0=\widetilde{T}_{j} A_{i}=2 \widehat{C}_{j} A_{i}-2 n_{i}=2-2 n_{i}$, we get $n_{i}=1$, $i=1, \ldots, 22$, and also $n_{21}^{\prime}=n_{22}^{\prime}=1$. So, we have

$$
2 \widetilde{T} \equiv \widetilde{T}_{1}+\widetilde{T}_{2}=2 \widehat{C}_{1}+2 \widehat{C}_{2}+\sum_{1}^{20} A_{i}+2 A_{21}+2 A_{22},
$$

where $\widetilde{T}$ is the pullback of a general hyperplane section of $\bar{X}_{40}$. This shows that $C_{1} \cup C_{2}$ contains 20 nodes of $\bar{X}_{40}$ which are 2-divisible. Analogously, the remaining 20 nodes of $\bar{X}_{40}$, contained in $C_{3} \cup C_{4}$, are also 2-divisible.

Proposition 8. The 40 nodes of a generic surface $X_{40}$ are 2-divisible.

Proof. This is immediate from Propositions 6 and 7 .

\subsection{Surfaces with $K_{S}^{2}=2 c_{2}=16$ and $q=4$}

From the previous section, for a surface $X_{40}$ with exactly 40 nodes as constructed above, there is a double covering $\pi: S \rightarrow X_{40}$ ramified over the nodes.

Proposition 9. We have $p_{g}(S)=5, q(S)=4$ and $K_{S}^{2}=16$.

Proof. The canonical line bundle $K_{S}$ is the pullback of $K_{X_{40}}$, which is nef; thus, $S$ is minimal and $K_{S}^{2}=2 K_{X_{40}}^{2}=16$.

Let $\widehat{X}_{40}$ be the smooth minimal model of $X_{40}$, let $A_{1}, \ldots, A_{40}$ be the $(-2)$-curves which contract to the nodes of $X_{40}$, and let $S^{\prime} \rightarrow \widehat{X}_{40}$ be the double covering with branch locus $\sum_{1}^{40} A_{i}$. The minimal model of $S^{\prime}$ is isomorphic to $S$.

Let $L$ be the divisor such that $\sum_{1}^{40} A_{i} \equiv 2 L$. The double covering formulas (see, for example, [BHPvdV04, Section V.22]) give

$$
\chi(S)=2 \chi\left(\widehat{X}_{40}\right)+\frac{1}{2} L\left(K_{\widehat{X}_{40}}+L\right)=12-10=2 .
$$

Let us compute $p_{g}(S)$. We have $p_{g}(S) \geqslant p_{g}\left(X_{40}\right)=5$, thus $q(S) \geqslant 4$. Suppose $q(S) \geqslant 5$. We know from [Deb82, Appendice] that one always has $p_{g}(S) \geqslant 2 q(S)-4$, with equality only if $S$ is the product of a curve of genus 2 and a curve of genus $q(S)-2 \geqslant 2$. Thus $p_{g}(S)=q(S)+1$ implies that $q(S)=5$ and $p_{g}(S)=6$, and $S$ is the product of a genus 2 curve with a genus 3 curve. The restriction of the canonical map of $S$ to a genus 2 fiber $F$ is a map of degree 2 to $\mathbb{P}^{1}$, the canonical map of $F$. Hence, the map $\left.\pi\right|_{F}$ is of degree at least 2 to $\mathbb{P}^{1}$. This gives a contradiction because, since $X_{40}$ is of general type, it is not a ruled surface.

Proposition 10. The surface $S$ is not covered by the bidisk $\mathbb{H} \times \mathbb{H}$. 


\section{Rito, X. Roulleau and A. Sarti}

Proof. If $S$ is universally covered by $\mathbb{H} \times \mathbb{H}$, then it is the quotient of $\mathbb{H} \times \mathbb{H}$ by a discrete cocompact subgroup $\Gamma$ of $\operatorname{Aut}(\mathbb{H} \times \mathbb{H})=\operatorname{Aut}(\mathbb{H})^{2} \rtimes(\mathbb{Z} / 2 \mathbb{Z})$ acting freely. Let

$$
\Gamma_{0}:=\Gamma \cap \operatorname{Aut}(\mathbb{H})^{2},
$$

and let $\Gamma_{0}^{\prime}$ and $\Gamma_{0}^{\prime \prime}$ be the projections of $\Gamma_{0}$ to the factors of $\operatorname{Aut}(\mathbb{H}) \times \operatorname{Aut}(\mathbb{H})$. By [Shi63, Theorem 1], if one of $\Gamma_{0}^{\prime}$ and $\Gamma_{0}^{\prime \prime}$ is discrete, so is the other. In that case, we say that $\Gamma$ is reducible.

If $\Gamma$ is irreducible, we know from [MS63, Introduction] that

$$
b_{1}\left(\mathbb{H} \times \mathbb{H} / \Gamma_{0}\right)=b_{1}\left(\mathbb{P}^{1} \times \mathbb{P}^{1}\right)=0 .
$$

This is impossible because $2 q=b_{1}$ and $q(S)=4$.

So, $\Gamma$ is reducible, and then $\Gamma_{0}$ is a finite index subgroup of $\Gamma_{0}^{\prime} \times \Gamma_{0}^{\prime \prime}$. It follows that $\mathbb{H} \times \mathbb{H} / \Gamma_{0}$ is a covering of the product of two curves $\mathbb{H} / \Gamma_{0}^{\prime} \times \mathbb{H} / \Gamma_{0}^{\prime \prime}$. We claim that $S$ is isogenous to a product of curves (that is, it is a quotient of a product of curves by a fixed-point-free group action). In fact, there exists a normal sublattice $\Gamma_{1}$ of $\Gamma_{0}$, of finite index, of the form

$$
\Gamma_{1}^{\prime} \times \Gamma_{1}^{\prime \prime} \subset \Gamma_{0} \subset \Gamma_{0}^{\prime} \times \Gamma_{0}^{\prime \prime} .
$$

This implies the existence of an étale map

$$
\mathbb{H} \times \mathbb{H} / \Gamma_{1}=\mathbb{H} / \Gamma_{1}^{\prime} \times \mathbb{H} / \Gamma_{1}^{\prime \prime} \longrightarrow \mathbb{H} \times \mathbb{H} / \Gamma_{0},
$$

the action being given by $\Gamma_{0} / \Gamma_{1}$.

Surfaces with $p_{g}=5$ and $q=4$ isogenous to a product of curves are classified in [BNP07]. They are of the form $(C \times H) /(\mathbb{Z} / 2 \mathbb{Z})$, where

a) $C$ and $H$ are curves of genus 3 with fixed-point-free involutions, or

b) $C$ is a curve of genus 5 with a fixed-point-free involution and $H$ is a bielliptic curve of genus 2 .

We know from [Pol06, Theorem 3.4] that the curves in case a) are hyperelliptic; hence, in both cases, the canonical map factors through a double covering of a ruled surface, and then the canonical image is not of general type. This implies that $S$ is not isogenous to a product of curves, giving a contradiction.

\subsection{The degeneration}

Proposition 11. The family of surfaces $S$ constructed above coincides with the family of surfaces constructed by Schoen in [Sch07].

Proof. The deformation space in Schoen's construction is locally irreducible (see Theorem 1); hence, we get from Proposition 2 that it suffices to show that the 4-dimensional family of surfaces $X_{40}$ degenerates to a 3-dimensional family of reducible surfaces which are each the union of a double quadric surface and a quartic Kummer surface, glued along a trope of the Kummer surface.

Let us consider the Igusa quartic given by the equation

$$
4\left(x^{4}+y^{4}+z^{4}+w^{4}+t^{4}+h^{4}\right)=\left(x^{2}+y^{2}+z^{2}+w^{2}+t^{2}+h^{2}\right)^{2},
$$

where $h:=-x-y-z-w-t$, in $\mathbb{P}^{4}(x, y, z, w, t)$. Recall from Section 3.2 that to a generic hyperplane section $H_{a, b, c, d}:=a x+b y+c z+d w-t$ of the Igusa quartic corresponds a quadric $Q_{a, b, c, d}$ such that $X_{40}:=I_{4} \cap Q_{a, b, c, d}$ (notice that the two $I_{4}$ that appear in Figure 1 denote 


\section{EXPLiCIT SCHOEN SURFACES}

isomorphic surfaces that are given by different equations in our computations). Let

$$
F_{a, b, c, d}=c_{1} x^{2}+\cdots+c_{15} w t, \quad c_{i}=c_{i}(a, b, c, d),
$$

be the defining polynomial of $Q_{a, b, c, d}$ in $\mathbb{P}^{4}$. The correspondence $H_{a, b, c, d} \mapsto Q_{a, b, c, d}$ is a rational map

$$
\varphi: \mathbb{A}^{4} \rightarrow \mathbb{P}^{14}=\mathbb{P}\left(H^{0}\left(\mathbb{P}^{4}, \mathcal{O}(2)\right)\right), \quad(a, b, c, d) \longmapsto\left(c_{1}: \cdots: c_{15}\right) .
$$

From our computations (see [Rit17]), if $H_{a, b, c, d}$ gives a trope of $I_{4}$, then $F_{a, b, c, d}$ vanishes identically. This happens, for instance, for $(a, b, c, d)=(0,0,-1,-1)$. We resolve the corresponding indeterminacy of $\varphi$ by blowing up: locally, this is done by evaluating the coefficients $c_{i}$ at $(a, a b, a c-1, a d-1)$. The computations give that

$$
F_{a, a b, a c-1, a d-1}=a^{3} \cdot G_{a, b, c, d}
$$

with $G$ of degree 2. Moreover, there exists a linear form $J_{b, c, d}$ such that

$$
G_{0, b, c, d}=x \cdot J_{b, c, d} .
$$

The hyperplane $\{x=0\}$ gives a trope of $I_{4}$ (a double quadric). For generic values of the parameters, the hyperplane given by $J_{b, c, d}$ is tangent to $I_{4}$ at a point (it gives a quartic Kummer surface), and the quadric given by $G_{a, b, c, d}$ meets $I_{4}$ at a 40 -nodal surface. One can verify from the equations obtained in [Rit17] that this quadric and the Kummer surface are glued along a trope of the Kummer surface.

\section{The surface $\bar{X}_{40}$ with $\Sigma_{5}$-symmetries}

In this section, we study a surface $\bar{S}$ which is the double cover of a particular 40-nodal, degree 8 , complete intersection surface with a large group of symmetries. Using these symmetries, we prove that its Picard number is maximal, and we find the isogeny class of its Albanese variety. We moreover describe another construction of a 3-dimensional subfamily of Schoen surfaces given as bidouble covers of some special Kummer surfaces.

\subsection{Some Schoen surfaces as bidouble covers}

Recall from Section 3.3 the complete intersection $\bar{X}_{40} \subset \mathbb{P}^{4}$ of the following quadric and quartic:

$$
\begin{gathered}
5\left(x^{2}+\cdots+t^{2}\right)-7(x+\cdots+t)^{2}=0, \\
4\left(x^{4}+\cdots+t^{4}+h^{4}\right)-\left(x^{2}+\cdots+t^{2}+h^{2}\right)^{2}=0,
\end{gathered}
$$

where $h=-(x+y+z+w+t)$. The surface $\bar{X}_{40}$ has 40 nodes (defined over the field $\mathbb{Q}(\sqrt{-15})$ ). The permutation group $\Sigma_{5}$ is a subgroup of $\operatorname{Aut}\left(\bar{X}_{40}\right)$, the automorphism group of $\bar{X}_{40}$.

Let $\bar{S} \rightarrow \bar{X}_{40}$ be the double cover branched over the 40 nodes, and let $\sigma$ be the corresponding involution of $\bar{S}$. Let $\hat{X}_{40}$ be the minimal resolution of $\bar{X}_{40}$. By the argument given in the proof of Proposition 6 , the integral cohomology group $H^{2}\left(\hat{X}_{40}, \mathbb{Z}\right)$ is torsion free. Thus, the NéronSeveri group $\operatorname{NS}\left(\hat{X}_{40}\right)$, which is a subgroup of $H^{2}\left(\hat{X}_{40}, \mathbb{Z}\right)$, is also torsion free. We also note that any automorphism in $\Sigma_{5}$ preserves the set of nodes. Then by Theorem 1e) in Section 1.3 of R. A. Livne's Ph.D. thesis, Harvard, 1981, each element of $\Sigma_{5}$ lifts to an automorphism of $\bar{S}$.

Proposition 12. Let $\tau \in \Sigma_{5}$ be a transposition. The quotient surface $Q:=\bar{X}_{40} / \tau$ is a $K 3$ surface with 15 nodes containing in the smooth locus two $(-2)$-curves $A_{16}$ and $A_{16}^{\prime}$ such that $A_{16} A_{16}^{\prime}=10$. The double cover $\bar{X}_{40} \rightarrow Q$ is branched over $A_{16}+A_{16}^{\prime}$. 


\section{Rito, X. Roulleau and A. Sarti}

Let $A_{1}, \ldots, A_{15}$ be the fifteen (-2)-curves in the resolution $\hat{Q}$ of $Q$. The divisors $A_{16}+\sum_{i=1}^{15} A_{i}$ and $A_{16}^{\prime}+\sum_{i=1}^{15} A_{i}$ are 2-divisible. The bidouble cover $\hat{S} \rightarrow \hat{Q}$ associated with the divisors

$$
D_{1}=\sum_{i=1}^{15} A_{i}, \quad D_{2}=A_{16}, \quad D_{3}=A_{16}^{\prime}
$$

gives the blow-up $\hat{S} \rightarrow \bar{S}$ at the 40 fixed points of $\sigma$; the bidouble cover decomposes as

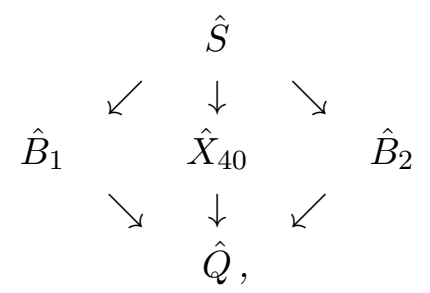

where $\hat{B}_{1}$ and $\hat{B}_{2}$ are Abelian surfaces $B_{1}$ and $B_{2}$ blown up at their 2-torsion points, each map $\hat{S} \rightarrow \hat{B}_{i}$ is a double cover branched over a curve of genus 4 and the maps $\hat{B}_{i} \rightarrow \hat{Q}$ for $i=1,2$ are branched over $D_{1}+D_{2}$, and $D_{1}+D_{3}$, respectively.

The group generated by the lifts of $\tau$ on $\bar{S}$ is $(\mathbb{Z} / 2 \mathbb{Z})^{2}$, and it contains $\sigma$.

Proof. Let $\tau \in \Sigma_{5}$ be a transposition (for example, the one exchanging the coordinates $x$ and $y$ ). Using Magma, we compute that the fixed-point set of $\tau$ is a union of two smooth genus 0 curves meeting at 10 points which are 10 nodes of $\bar{X}_{40}$. Moreover, the quotient of the surface $\bar{X}_{40}$ by $\tau$ is a quartic $K 3$ surface $Q \hookrightarrow \mathbb{P}^{3}$ which has 15 nodes (see [Rit17, Section D]).

The image of the fixed-point set of $\tau$ by the quotient map is $A_{16}+A_{16}^{\prime}$, where $A_{16}$ and $A_{16}^{\prime}$ are two $(-2)$-curves which are disjoint from the 15 nodes and meet transversally at 10 points. It is the intersection of $Q$ with a quadric in $\mathbb{P}^{3}$.

Let $A_{1}, \ldots, A_{15}$ be the fifteen (-2)-curves above the nodes on the minimal resolution $\hat{Q}$ of $Q$. Let us keep the same notation for the strict transforms of $A_{16}$ and $A_{16}^{\prime}$ on $\hat{Q}$. The 16 curves $A_{1}, \ldots, A_{15}, A_{16}$ are disjoint, and so are the 16 curves $A_{1}, \ldots, A_{15}, A_{16}^{\prime}$. Thus by [Nik75, Theorem 1], the divisors $A_{16}+\sum_{i=1}^{15} A_{i}$ and $A_{16}^{\prime}+\sum_{i=1}^{15} A_{i}$ are 2-divisible. Using the 3 divisors $D_{1}$, $D_{2}, D_{3}$, the associated bidouble cover $\hat{S} \rightarrow \hat{Q}$ gives the blow-up of $\bar{S}$ at the 40 fixed points of $\sigma$ (see [Par91] or [Cat99] for information on bidouble covers); the remaining assertions follow.

Remark 13. More generally, one can prove that there exists a 3-dimensional family of quartic $K 3$ surfaces with 15 nodes, containing on their smooth locus two (-2)-curves $A_{16}$ and $A_{16}^{\prime}$ such that $A_{16} A_{16}^{\prime}=10$ (cf. [Rem07, Pia23]). Their associated bidouble covers as above give a 3 -dimensional subfamily of Schoen surfaces.

It is interesting to compare this construction of Schoen surfaces by bidouble covers with the construction of Lagrangian surfaces done by Bogomolov and Tschinkel in [BT00, Sections 3 and 4].

\subsection{The 240 automorphisms of $\bar{S}$}

We will use standard results in representation theory for which we refer the reader to [FH91]. The permutation group $\Sigma_{5}$ has seven irreducible representations (up to isomorphism), which we denote by

$$
U, \quad U^{\prime}, V, \quad V^{\prime}=V \otimes U^{\prime}, \quad W, \quad W^{\prime}=W \otimes U^{\prime}, \quad \wedge^{2} V
$$




\section{EXPLICIT SCHOEN SURFACES}

of respective dimension $1,1,4,4,5,5,6$, where $U$ is the trivial representation, $U^{\prime}$ is the signature, the 4-dimensional representation $V$ satisfies $\operatorname{Tr}(\tau)=2$, and the 5-dimensional representation $W$ is determined by $\operatorname{Tr}(\tau)=1$ ( $\operatorname{Tr}$ is the trace and $\tau \in \Sigma_{5}$ is a transposition).

One has $K_{\bar{X}_{40}}=\mathcal{O}(1)$. By looking at the symmetries of the equations of $\bar{X}_{40}$, we see that the representation of $\Sigma_{5}$ on $H^{0}\left(\bar{X}_{40}, K_{\bar{X}_{40}}\right)$ is faithful. On $\mathbb{P}^{4}$, the point $(1: 1: 1: 1: 1)$ is invariant; thus, the corresponding vector space is stable, and the representation is not irreducible. The only non-irreducible 5-dimensional faithful representations are

$$
U+V, \quad U+V^{\prime}, \quad U^{\prime}+V, \quad U^{\prime}+V^{\prime} .
$$

Let $\operatorname{Aut}(\bar{S})^{o}$ be the subgroup of $\operatorname{Aut}(\bar{S})$ generated by the lifts of the elements of $\Sigma_{5} \subset$ $\operatorname{Aut}\left(\bar{X}_{40}\right)$. There is a natural exact sequence

$$
0 \rightarrow \mathbb{Z} / 2 \mathbb{Z} \rightarrow \operatorname{Aut}(\bar{S})^{o} \rightarrow \Sigma_{5} \rightarrow 0,
$$

where the morphism $\mathbb{Z} / 2 \mathbb{Z} \rightarrow \operatorname{Aut}(\bar{S})^{o}$ is obtained by the inclusion of $\sigma$. By Schur theory, the group extensions $0 \rightarrow \mathbb{Z} / 2 \mathbb{Z} \rightarrow H \rightarrow \Sigma_{5} \rightarrow 0$ of $\Sigma_{5}$ by $\mathbb{Z} / 2 \mathbb{Z}$ are classified by the second homology group $H^{2}\left(\Sigma_{5}, \mathbb{Z} / 2 \mathbb{Z}\right)$, which is isomorphic to $(\mathbb{Z} / 2 \mathbb{Z})^{2}$; therefore, $\operatorname{Aut}(\bar{S})^{o}$ is one of the groups

$$
\mathbb{Z} / 2 \mathbb{Z} \times \Sigma_{5}, \quad 2 . \Sigma_{5}^{-}, \quad 2 . \Sigma_{5}^{+}, \quad 4 . A_{5},
$$

which we will describe later.

Theorem 14. The group $\operatorname{Aut}(\bar{S})^{o}$ is $2 . \Sigma_{5}^{+}$.

We prove this result by showing that $\operatorname{Aut}(\bar{S})^{o}$ cannot be $\mathbb{Z} / 2 \mathbb{Z} \times \Sigma_{5}, 2 . \Sigma_{5}^{-}$or $4 . A_{5}$. We need the following lemma.

Lemma 15. The trace of the involution $\sigma$ on $H^{0}\left(\bar{S}, \Omega_{\bar{S}}\right)$ is -4 .

Proof. The minimal resolution $\hat{X}_{40}$ of the quotient surface $\bar{S}=\bar{X}_{40} / \sigma$ is regular. By [Bea96, Lemma VI.11 and Example VI.12 3)], the space of $\sigma$-invariant 1-forms on $\bar{S}$ and the space of 1-forms on $\hat{X}_{40}$ have the same dimension. Therefore, $\sigma$ acts on $H^{0}\left(\bar{S}, \Omega_{\bar{S}}\right)$ by multiplication by -1 ; the result follows.

We moreover remark that the morphism

$$
\varphi_{2,0}: \wedge^{2} H^{0}\left(\bar{S}, \Omega_{\bar{S}}\right) \longrightarrow H^{0}\left(\bar{S}, K_{\bar{S}}\right) \simeq H^{0}\left(\bar{X}_{40}, K_{\bar{X}_{40}}\right)
$$

is equivariant under $\operatorname{Aut}(\bar{S})^{o}$, and we know that it has a 1-dimensional kernel (since it is a Schoen surface). The group $\operatorname{Aut}(\bar{S})^{o}$ acts on $H^{0}\left(\bar{S}, K_{\bar{S}}\right)=H^{0}\left(\bar{X}_{40}, K_{\bar{X}_{40}}\right)$ through $\operatorname{Aut}(\bar{S})^{o} / \sigma=\Sigma_{5}$.

Proof of Theorem 14. Suppose that we have $\operatorname{Aut}(\bar{S})^{o}=\mathbb{Z} / 2 \mathbb{Z} \times \Sigma_{5}$. If the 4-dimensional representation $H^{0}\left(\bar{S}, \Omega_{\bar{S}}\right)$ of $\Sigma_{5}$ is faithful, then it is $V$ or $V^{\prime}$, and $\wedge^{2} H^{0}\left(\bar{S}, \Omega_{\bar{S}}\right)=\wedge^{2} V=\wedge^{2} V^{\prime}$ is an irreducible (6-dimensional) representation; this gives a contradiction. Therefore, $H^{0}\left(\bar{S}, \Omega_{\bar{S}}\right)=$ $U^{a}+U^{\prime b}$, but then the representation of $\Sigma_{5}=\operatorname{Aut}(\bar{S})^{o} / \sigma$ on $\wedge^{2} H^{0}\left(\bar{S}, \Omega_{\bar{S}}\right)$ is not faithful, which again gives a contradiction.

The group $2 . \Sigma_{5}^{-}$is group number 89 among the order 240 groups in the Magma database. It contains a unique involution. But by Proposition 12, the automorphisms of $\bar{S}$ lifting the transpositions of $\Sigma_{5}$ acting on $\bar{X}_{40}$ are involutions; thus, Aut $(\bar{S})^{o}$ cannot be $2 . \Sigma_{5}^{-}$.

The group $A_{5} .4$ (group number 91 in the Magma database) has 14 irreducible representations $\chi_{i}$, for $i=1, \ldots, 14$, of respective dimensions $1^{4}, 4^{4}, 5^{4}$ and $6^{2}$, where $a^{b}$ means $a$ repeated $b$ times. 


\section{Rito, X. Roulleau and A. Sarti}

Let $W_{4}$ be a non-irreducible 4-dimensional representation of $A_{5} .4$. It is easy to see that $\wedge^{2} W_{4}$ is not a faithful representation of $\Sigma_{5}=\operatorname{Aut}(\bar{S})^{o} / \sigma$; thus, $\wedge^{2} H^{0}\left(\bar{S}, \Omega_{\bar{S}}\right)$ cannot be such a representation $W_{4}$.

Looking at the character table (for instance given by Magma), we see that the representation $H^{0}\left(\bar{S}, \Omega_{\bar{S}}\right)$ cannot be $\chi_{5}$ or $\chi_{6}$ since the trace of the involution $\sigma$ must be -4 . The two remaining 4-dimensional representations $\chi_{7}$ and $\chi_{8}$ satisfy $\wedge^{2} \chi_{7}=\wedge^{2} \chi_{8}=\chi_{14}$ (for the computation of the wedge product of a representation, see [FH91]), which is an irreducible representation of $\Sigma_{5}=\operatorname{Aut}(\bar{S})^{o} / \sigma$; thus, $A_{5} .4$ is not $\operatorname{Aut}(\bar{S})^{o}$. The only possibility is thus $\operatorname{Aut}(\bar{S})^{o}=2 . \Sigma_{5}^{+}$.

\subsection{The group $2 . \Sigma_{5}^{+}$and its action on $\bar{S}$}

The group 2. $\Sigma_{5}^{+}$(number 90 among groups of order 240 in the Magma database) has 12 irreducible representations $\chi_{1}, \ldots, \chi_{12}$, of respective dimensions $1^{2}, 4^{5}, 5^{2}$ and $6^{3}$. It has 21 involutions, divided into two conjugacy classes, one containing a unique element $\sigma$, which is the involution of the double cover $\bar{S} \rightarrow \bar{X}_{40}$. Since the trace of $\sigma$ on $\chi_{3}$ and $\chi_{5}$ is not -4 , the only possibilities are $H^{0}\left(\bar{S}, \Omega_{\bar{S}}\right)=\chi_{4}, \chi_{6}$ or $\chi_{7}$. One has

$$
\wedge^{2} \chi_{4}=\chi_{1}+\chi_{2}+\chi_{3} \text { and } \wedge^{2} \chi_{6}=\wedge^{2} \chi_{7}=\chi_{2}+\chi_{9}
$$

The representation of $2 . \Sigma_{5}^{+}$on $\chi_{9}$ gives an irreducible 5-dimensional representation of $2 . \Sigma_{5}^{+} / \sigma=$ $\Sigma_{5}$, which is impossible since $H^{0}\left(\bar{X}_{40}, K_{\bar{X}_{40}}\right)$ is not irreducible. We have thus proved that $H^{0}\left(\bar{S}, \Omega_{\bar{S}}\right)=\chi_{4}$, which has character

$$
\begin{array}{ccccccccccccc}
\text { Order } & 1 & 2 & 2 & 3 & 4 & 5 & 6 & 6 & 6 & 8 & 8 & 10 \\
\text { Trace } & 4 & -4 & 0 & -2 & 0 & -1 & 0 & 0 & 2 & 0 & 0 & 1
\end{array}
$$

We conclude the following.

Proposition 16. The representation of the group $2 . \Sigma_{5}^{+}$on $H^{0}\left(\bar{S}, \Omega_{\bar{S}}\right)$ is $\chi_{4}$. Moreover, one has $\wedge^{2} H^{0}\left(\bar{S}, \Omega_{\bar{S}}\right)=\chi_{1}+\chi_{2}+\chi_{3}$ and $H^{1,1}(A)=\chi_{4} \otimes \chi_{4}=\chi_{1}+\chi_{2}+\chi_{3}+\chi_{5}+\chi_{10}$, where $A$ is the Albanese variety of $\bar{S}$.

The group $\Sigma_{5}=\operatorname{Aut}(\bar{S})^{o} / \sigma$ acts on $\wedge^{2} \chi_{4}$ and $\wedge^{2} \chi_{4}=U+U^{\prime}+V$.

Proposition 17. The representation of $2 . \Sigma_{5}^{+}$on $H^{0}\left(\bar{S}, K_{\bar{S}}\right)$ is $\chi_{2}+\chi_{3}$.

Proof. The trace of an involution $\iota \neq \sigma$ in $2 . \Sigma_{5}^{+}$acting on $\chi_{4}$ equals 0 ; thus, the eigenvalues of $\iota$ on the space of holomorphic 1 -forms are $1,1,-1,-1$. Moreover, since $\wedge^{2} \chi_{4}=\chi_{1}+\chi_{2}+\chi_{3}$, the involution $\iota$ acts on $H^{0}\left(\bar{S}, K_{\bar{S}}\right)$ with trace -1 or -3 according to whether

$$
H^{0}\left(\bar{S}, K_{\bar{S}}\right)=U+V \quad \text { or } \quad H^{0}\left(\bar{S}, K_{\bar{S}}\right)=U^{\prime}+V .
$$

Then the eigenvalues of $\iota$ on $H^{0}\left(\bar{S}, K_{\bar{S}}\right)$ are, respectively, $1,1,-1,-1,-1$ and $1,-1,-1,-1,-1$. By [Bea96, Lemma VI.11 and Example VI.12,3)], the quotient surface has invariants $q=2$ and $p_{g}=2$ or $p_{g}=1$, respectively. By Proposition 12, that quotient surface is (birational to) an Abelian surface, and it is the second case that is actually occurring; thus, $H^{0}\left(\bar{S}, K_{\bar{S}}\right)=U^{\prime}+V$, which corresponds to the representation $\chi_{2}+\chi_{3}$ for $2 . \Sigma_{5}^{+}$.

There is a basis $\omega_{1}, \ldots, \omega_{4}$ of $H^{0}\left(\bar{S}, \Omega_{\bar{S}}\right)$ such that the action of $2 . \Sigma_{5}^{+}$is generated by the following matrices of order 2 and 8 :

$$
\left(\begin{array}{cccc}
0 & 1 & 0 & 0 \\
1 & 0 & 0 & 0 \\
0 & 0 & 0 & 1 \\
0 & 0 & 1 & 0
\end{array}\right), \quad\left(\begin{array}{cccc}
\frac{1}{2} \sqrt{2}(1+I) & 0 & -\frac{1}{2} \sqrt{2}(1+I) & -I \\
0 & 0 & -1 & 0 \\
0 & 1 & -\sqrt{2} & -1 \\
0 & 0 & 0 & \frac{1}{2} \sqrt{2}(1-I)
\end{array}\right)
$$




\section{EXPLICIT SCHOEN SURFACES}

where $I^{2}=-1$. We have $\wedge^{2} H^{0}\left(\bar{S}, \Omega_{\bar{S}}\right)=\chi_{1}+\chi_{2}+\chi_{3}$, where the trivial representation $\chi_{1}$ is generated by the indecomposable vector $v=\omega_{1} \wedge \omega_{4}+\omega_{2} \wedge \omega_{3}$, which generates the kernel of $\wedge^{2} H^{0}\left(\bar{S}, \Omega_{\bar{S}}\right) \rightarrow H^{0}\left(\bar{S}, K_{\bar{S}}\right)$. By the theorem of Castelnuovo-De Franchis, this gives another proof that $\bar{S}$ has no fibration onto a curve of genus at least 2 .

\subsection{The periods of the Albanese variety of $\bar{S}$}

Let us study the Albanese variety of $\bar{S}$.

Proposition 18. The Albanese variety $A$ of $\bar{S}$ is isogenous to $E^{4}$, where $E$ is an elliptic curve with complex multiplication by $\mathbb{Z}[\sqrt{-15}]$.

Proof. The Albanese variety $A$ of $\bar{S}$ is $A=H^{0}\left(\bar{S}, \Omega_{\bar{S}}\right)^{*} / \Lambda$, where $\Lambda=H_{1}(\bar{S}, \mathbb{Z}) \subset H^{0}\left(\bar{S}, \Omega_{\bar{S}}\right)^{*}$. Since $2 . \Sigma_{5}^{+}$acts on $A$, the lattice $\Lambda$ is a $2 . \Sigma_{5}^{+}$-stable lattice in $\chi_{4}=H^{0}\left(\bar{S}, \Omega_{\bar{S}}\right)^{*}$. The representation $\chi_{4}$ has Schur index 2, and one computes that there exists a non-trivial $2 . \Sigma_{5}^{+}$-invariant antisymmetric bilinear form on $V_{4}=\chi_{4}$. By [PZ06, Theorem 4.1(ii $\left.)_{4}\right)$, this implies that $A$ is isogenous to $E^{4}$, where $E$ is an elliptic curve with $\mathrm{CM}$.

Let $\tau$ be the involution acting on $\bar{X}_{40}$ by exchanging the first two coordinates. The line

$$
L=\left\{X+Z=Y+\frac{1}{4}(-1+I \sqrt{15}) W=0\right\}, \quad I^{2}=-1,
$$

is contained in the quotient surface $Q=\bar{X}_{40} / \tau$, the equation of which is given in [Rit17]. This line contains three nodes $a_{1}, a_{2}, a_{3}$ and cuts the two $(-2)$-curves disjoint from the 15 nodes in points denoted by $a_{4}$ and $a_{5}$. By Proposition 12 and its proof, the surface $Q$ is birational to two Kummer surfaces $B_{i} /[-1]$, for $i=1,2$, where each $B_{i}$ is an Abelian surface. The four points $a_{1}, \ldots, a_{4}$ are the branch points of a degree 2 cover $E \rightarrow L$, where $E$ is therefore an elliptic curve on $B_{1}$ (say). The line $L$ is also the image of an elliptic curve on $B_{2}$, with branch points $a_{1}, a_{2}$, $a_{3}, a_{5}$. Using the cross ratio for the points $a_{1}, \ldots, a_{4}$, one finds that

$$
E=\left\{y^{2}=x(x-1)(x-\lambda)\right\},
$$

where

$$
\lambda=\frac{1}{64}(17+21 \sqrt{5}+I(7 \sqrt{15}-17 \sqrt{3})), \quad I^{2}=-1 .
$$

The $j$-invariant of $E$ is

$$
-\frac{3^{3} 5}{2}\left(5 \cdot 283+7^{2} 13 \sqrt{5}\right)
$$

and using Magma again (see [Rit17, Section E]), one obtains that $E$ has CM by the order $\mathbb{Z}[\sqrt{-15}]$ (taking the cross ratio for $a_{1}, a_{2}, a_{3}, a_{5}$ gives an elliptic curve with $j$-invariant conjugated to $j(E)$ and CM by the same order). We know by Proposition 12 that $\bar{S}$ admits a map onto $B_{1}$, giving the result.

\subsection{The surface $\bar{S}$ has maximal Picard number}

Finally, we prove the following.

Theorem 19. The surface $\bar{S}$ and the minimal resolution $\hat{X}_{40}$ of $\bar{X}_{40}$ have maximal Picard number, equal to, respectively, 12 and 52.

Proof. The Albanese variety $A$ of $\bar{S}$ is isogeneous to $E^{4}$, where $E$ is an elliptic curve with CM; therefore, $A$ has maximal Picard number. Moreover, the map

$$
H^{2,0}(A)=\wedge^{2} H^{0}\left(\bar{S}, \Omega_{\bar{S}}\right) \longrightarrow H^{2,0}(\bar{S})=H^{0}\left(\bar{S}, K_{\bar{S}}\right)
$$




\section{Rito, X. Roulleau and A. Sarti}

is surjective; thus, by [Bea14, Proposition 2(a)], the surface $\bar{S}$ has maximal Picard number. There is a dominant rational map $\bar{S} \rightarrow \hat{X}_{40}$, and $\bar{S}$ has maximal Picard number; thus, by [Bea14, Proposition 2(b)], the surface $\hat{X}_{40}$ has maximal Picard number. It is easy to check that $h^{1,1}(\bar{S})=12$ and $h^{1,1}\left(\hat{X}_{40}\right)=52$.

\section{Appendix. Quartics with 15 nodes}

Let $Q_{15}$ be a $K 3$ surface in $\mathbb{P}^{3}(\mathbb{C})$ with 15 nodes. In this section we show the following:

- The moduli space of quartic $K 3$ surfaces with 15 nodes can be described as the moduli space of $K 3$ surfaces polarized by some lattice $N$ that we describe below, and it is irreducible.

- A generic $K 3$ surface with 15 nodes can be realized as a section of the Igusa quartic threefold, generalizing a similar result for Kummer quartic surfaces.

The K3 surfaces as $Q_{15}$ above are described in [GS16, Theorem 8.6] and in [Gar17, Section 5]. We recall here the following result for convenience.

TheOREM 20 ([GS16]). Let $\tilde{Q}_{15}$ be a projective $K 3$ surface with 15 disjoint smooth rational curves $M_{i}$, for $i=1, \ldots, 15$.

1) The Néron-Severi group of $\tilde{Q}_{15}$ contains the lattice $M_{(\mathbb{Z} / 2 \mathbb{Z})^{4}}$ (which is the smallest primitive sublattice of the $K 3$ lattice containing the 15 rational curves $\left.M_{i}\right)$.

2) There exists a $K 3$ surface $X$ with a symplectic action by $G=(\mathbb{Z} / 2 \mathbb{Z})^{4}$ such that $\tilde{Q}_{15}$ is the minimal resolution of the quotient $X / G$.

With the same notation as in Theorem 20, assume that $\tilde{Q}_{15}$ is the minimal resolution of $Q_{15}$. By [GS16, Theorem 8.3], the Néron-Severi group $\operatorname{NS}\left(\tilde{Q}_{15}\right)$ contains the sublattice $\langle 4\rangle \oplus\langle-2\rangle^{\oplus 15}$ of rank 16. We denote by $M_{1}, \ldots, M_{15}$ the fifteen $(-2)$-curves that are the exceptional divisors on $\tilde{Q}_{15}$. In Section A.1, we show that $\operatorname{NS}\left(\tilde{Q}_{15}\right)$ must contain a special overlattice of $\langle 4\rangle \oplus\langle-2\rangle^{\oplus 15}$, which is described in details in [GS16, Theorem 8.3] and is generated by

- a pseudo-ample class $L$ with $L^{2}=4$ (and $L \cdot M_{i}=0$ for $i=1, \ldots, 15$ ),

- the lattice $M:=M_{(\mathbb{Z} / 2 \mathbb{Z})^{4}}$ (that we recall below),

- a class $(L-v) / 2$, where $v$ contains exactly six of the $M_{i}$ in its support (these are not chosen arbitrarily; we recall them below).

\section{A.1 The lattice $M$ and the class $v$}

The lattice $M$ has discriminant $2^{7}$, and it is described by Nikulin [Nik76, $\S 7$. Let $K$ denote the Kummer lattice, that is, the smallest primitive sublattice of the $K 3$ lattice that contains sixteen (-2)-classes. This is negative definite and has rank 16 and discriminant $2^{6}$; see [Nik75]. We identify the 16 classes of the Kummer lattice with the elements of $(\mathbb{Z} / 2 \mathbb{Z})^{4}$, so we denote the curves by $K_{i j k h}$ with $i, j, k, h \in\{0,1\}$. One can identify $M=K_{0000}^{\perp} \cap K$. By using the description of $K$ (see, for example, [GS16]), we see that the following hold:

- The 15 classes $K_{i j k h}$ with $(i, j, k, h) \in(\mathbb{Z} / 2 \mathbb{Z})^{4} \backslash\{(0,0,0,0)\}$ are contained in $M$.

- Let $W$ be an hyperplane in the affine space $(\mathbb{Z} / 2 \mathbb{Z})^{4}$ with an equation $\sum_{i=1}^{4} \alpha_{i} x_{i}=1$, with $\alpha_{i} \in\{0,1\}$. Then the 15 classes $(1 / 2) \sum_{p \in W} K_{p}$ are contained in $M$. Each of these classes contains exactly eight distinct (-2)-classes of the $K_{i j k h}$. 


\section{EXPLICIT SCHOEN SURFACES}

Finally, as explained in [GS16, Theorem 8.3], the class $v$ such that $(L-v) / 2 \in \mathrm{NS}(Y)$ can be taken as the sum

$$
K_{0001}+K_{0010}+K_{0011}+K_{1000}+K_{0100}+K_{1100} .
$$

Notation. For the rest of the section, we will denote the fifteen $(-2)$-classes by $M_{i}$ with $i=$ $1, \ldots, 15$ or by $K_{i j k h}$ with $(i, j, k, h) \in(\mathbb{Z} / 2 \mathbb{Z})^{4} \backslash\{(0,0,0,0)\}$, depending on whether or not it is important to specify the indices.

\section{A.2 The Néron-Severi group}

Let $N$ denote the abstract lattice generated by $\mathbb{Z} L \oplus M$ and by a class $(L-v) / 2$. The following result is contained in the paper [Gar17, Proposition 5.1] in a more general context; for convenience, we give here a specific proof for our situation.

Proposition 21. Let $\tilde{Q}_{15}$ be the minimal resolution of a $K 3$ quartic surface with 15 nodes. Then $\tilde{Q}_{15}$ is pseudo-ample $N$-polarized; that is, there is a primitive embedding of $N$ in $\operatorname{NS}\left(\tilde{Q}_{15}\right)$, and the image of $N$ in $\operatorname{NS}\left(\tilde{Q}_{15}\right)$ contains a pseudo-ample class.

Proof. We use a similar argument as in the proof of [GS16, Theorem 8.6]. By construction and by [GS16, Theorem 8.6,1)], we know that $\mathbb{Z} L \oplus M$ is a sublattice of $\operatorname{NS}\left(\tilde{Q}_{15}\right)$ (and $L$ is pseudoample). Let $Q$ be the orthogonal complement of $\mathbb{Z} L \oplus M$ in $\operatorname{NS}\left(\tilde{Q}_{15}\right)$, and let $R:=(\mathbb{Z} L \oplus M) \oplus Q$. Then $\operatorname{NS}\left(\tilde{Q}_{15}\right)$ is an overlattice of finite index of $R$, and $R^{\vee} / R$ has number of generators $\ell(R)$ equal to $1+7+\ell(Q)$, where $\ell(Q)$ denotes the number of generators of $Q^{\vee} / Q$ (recall that $M$ has discriminant $2^{7}$ and discriminant group isomorphic to $\left.(\mathbb{Z} / 2 \mathbb{Z})^{7}\right)$. If $k$ denotes the index of $R$ in $\operatorname{NS}\left(\tilde{Q}_{15}\right)$, then we have

$$
\ell(\mathrm{NS}(Y))=8+\ell(Q)-2 k .
$$

Let $T_{\tilde{Q}_{15}}$ be the transcendental lattice. Since the $K 3$ lattice is unimodular, we have

$$
\ell\left(\operatorname{NS}\left(\tilde{Q}_{15}\right)\right)=\ell\left(T_{\tilde{Q}_{15}}\right) \leqslant \operatorname{rk}\left(T_{\tilde{Q}_{15}}\right)=22-\operatorname{rk}\left(\operatorname{NS}\left(\tilde{Q}_{15}\right)\right)=6-\operatorname{rk}(Q) .
$$

This gives $8+\ell(Q)-2 k \leqslant 6-\operatorname{rk}(Q)$ and then

$$
k \geqslant \frac{1}{2}(\ell(Q)+\operatorname{rk}(Q))+1 .
$$

Observe that $k$ is the minimum number of classes we have to add to $R$ to obtain the lattice $\operatorname{NS}\left(\tilde{Q}_{15}\right)$. The classes can be of two types: either they are classes in $(\mathbb{Z} L \oplus M)^{\vee} /(\mathbb{Z} L \oplus M)$, or they are sums $\nu+\nu^{\prime}$ with $\nu \in(\mathbb{Z} L \oplus M)^{\vee} /(\mathbb{Z} L \oplus M)$ and $\nu^{\prime} \in Q^{\vee} / Q$. The maximum number of classes of the second kind is bounded by $\ell(Q)$, so we must have at least $(\operatorname{rk}(Q)-\ell(Q)) / 2+1$ classes of the first type. Since $\operatorname{rk}(Q)-\ell(Q) \geqslant 0$, we have at least one class of the first kind, that is, contained in $(\mathbb{Z} L \oplus M)^{\vee} /(\mathbb{Z} L \oplus M)$. The discriminant group here is $\mathbb{Z} / 4 \mathbb{Z} \oplus M^{\vee} / M=\mathbb{Z} / 4 \mathbb{Z} \oplus(\mathbb{Z} / 2 \mathbb{Z})^{7}$. Observe that, here, a class $\nu$ is then of the form $(a L / 4+w / 2)$, and we have $2(a L / 4+w / 2)-w \in \operatorname{NS}(Y)$ so that $a= \pm 2$. This shows that the class can be assumed to be $(L+w) / 2$. Moreover, the square of this class must be in $2 \mathbb{Z}$, which gives $L^{2}+w^{2}=0 \bmod 8$. If $h$ is the number of curves contained in the support of $w$, we get $2-h=0 \bmod 4$. By the description of the discriminant group of $M$ [GS16, Proposition 8.2], we get that $h=6$ or $h=10$, so that we may assume that the class is of the form $(L-v) / 2$ as in the statement (since by [GS16, Proposition 8.2], if we take a class with $h=10$, we get the same lattice $N)$. This concludes the proof.

Remark 22. One can easily show that if a $K 3$ surface has Néron-Severi group exactly isometric to $N$, then it admits a projective model as a quartic surface with 15 nodes (that is, $N$ contains 


\section{Rito, X. Roulleau and A. Sarti}

a pseudo-ample class), so the corresponding moduli space $X_{\Gamma}$ is 4-dimensional, and (see [Hun96, Section 2.3]) it is an arithmetic quotient by some subgroup $\Gamma$ of the isometries of the $K 3$ lattice of the domain

$$
\mathcal{D}_{N}=\left\{\omega \in \mathbb{P}(T \otimes \mathbb{C}) \mid \omega^{2}=0, \omega \bar{\omega}=0\right\},
$$

where $T$ is the orthogonal complement of $N$ in the $K 3$ lattice $U^{3} \oplus E_{8}(-1)^{2}$. This has rank 4, and it is the transcendental lattice of the generic $K 3$ surface in the family.

\section{A.3 The moduli space}

Let $\mathcal{M}_{N}$ be the moduli space of $K 3$ surfaces that are pseudo-ample $N$-polarized. This moduli space is described, for example, in [Dol96, Section 1], where it is shown that it is isomorphic to the space $X_{\Gamma}$ from Remark 22 .

Proposition 23. The moduli space $\mathcal{M}_{N}$ is irreducible.

Proof. The embedding of $N$ into the $K 3$ lattice is unique by [Nik80, Theorem 1.14 .4 and Remark 1.14.5] (see also [GS16, Theorem 8.3]). By the construction of [Dol96, Section 3], the domain $\mathcal{D}_{N}$ has two connected components, both isomorphic to a bounded Hermitian domain of type $\mathrm{IV}_{19-(\operatorname{rk}(N)-1)}=\mathrm{IV}_{4}$. Observe that by [Nik80, Theorems 1.13.2 and 1.14.2], the orthogonal complement of $N$ in the $K 3$ lattice is uniquely determined by the signature and discriminant form. We compute, as in [GS16, Theorem 8.3], that the discriminant group of $N$ is $(\mathbb{Z} / 4 \mathbb{Z}) \oplus(\mathbb{Z} / 2 \mathbb{Z})^{5}$. If we denote by $q_{2}$ the discriminant form of the lattice $U(2)$ (this is the lattice $U$ with the bilinear form multiplied by 2), then the discriminant form is the same as $q_{2} \oplus q_{2}$ on $(\mathbb{Z} / 2 \mathbb{Z})^{4}$ and takes value $1 / 4$ and $1 / 2$ on the remaining part $(\mathbb{Z} / 4 \mathbb{Z}) \oplus(\mathbb{Z} / 2 \mathbb{Z})$. Hence, we can identify $N^{\perp}$ (modulo isometries) with the lattice

$$
U(2) \oplus U(2) \oplus\langle-2\rangle \oplus\langle-4\rangle .
$$

By [Dol96, Proposition 5.6 and Lemma 5.4], there is an involution in $\Gamma$ that exchanges the two connected components of $\mathcal{D}_{N}$, so that $X_{\Gamma} \simeq \mathcal{M}_{N}$ is irreducible.

Since the hyperplane sections of the Igusa quartic give a 4-dimensional family of quartic surfaces with 15 nodes, Proposition 23 implies the following.

TheOREm 24. A generic quartic K3 surface with 15 nodes can be realized as a section of the Igusa quartic.

Remark 25. An interesting locus in the moduli space $\mathcal{M}_{N}$ corresponds to quartic Kummer surfaces with 16 nodes that can be described as tangent sections of the Igusa quartic; see [Hun96, Chapter 3, Section 3.3.3].

\section{ACKNOWLEDGEMENTS}

The first author thanks the university of Poitiers for the hospitality during his visit in March 2016. The authors would like to thank Amir Dzambic, Alice Garbagnati, Margarida Mendes Lopes, Rita Pardini, Pierre Py and Chad Schoen for useful conversations or correspondence.

\section{REFERENCES}

Bea79 A. Beauville, L'application canonique pour les surfaces de type général, Invent. Math. $\mathbf{5 5}$ (1979), no. 2, 121-140; doi:10.1007/BF01390086. 


\section{EXPLICIT SCHOEN SURFACES}

Bea96 Complex algebraic surfaces, 2nd edn, London Math. Soc. Stud. Texts, vol. 34 (Cambridge Univ. Press, Cambridge, 1996); doi:10.1017/CB09780511623936.

Bea13 A tale of two surfaces, 2013, arXiv:1303.1910.

Bea14_ Some surfaces with maximal Picard number, J. Éc. Polytech. Math. 1 (2014), 101116; doi:10.5802/jep.5.

BHPvdV04 W.P. Barth, K. Hulek, C. A. M. Peters and A. van de Ven, Compact complex surfaces, 2nd edn, Ergeb. Math. Grenzgeb. (3), vol. 4 (Springer-Verlag, Berlin, 2004); doi:10.1007/ 978-3-642-57739-0.

BNP07 M. A. Barja, J. C. Naranjo and G.P. Pirola, On the topological index of irregular surfaces, J. Algebraic Geom. 16 (2007), no. 3, 435-458; doi:10.1090/S1056-3911-06-00454-1.

BPS10 F. Bastianelli, G. P. Pirola and L. Stoppino, Galois closure and Lagrangian varieties, Adv. Math. 225 (2010), no. 6, 3463-3501, doi:10.1016/j.aim.2010.06.006.

BT00 F. Bogomolov and Y. Tschinkel, Lagrangian subvarieties of abelian fourfolds, Asian J. Math. 4 (2000), no. 1, 19-36; doi:10.4310/AJM.2000.v4.n1.a3.

Cam95 F. Campana, Remarques sur les groupes de Kähler nilpotents, Ann. Sci. École Norm. Sup. (4) 28 (1995), no. 3, 307-316; doi:10.24033/asens. 1715.

Cat99 F. Catanese, Singular bidouble covers and the construction of interesting algebraic surfaces, Algebraic Geometry: Hirzebruch 70 (Warsaw, 1998), Contemp. Math., vol. 241 (Amer. Math. Soc., Providence, RI, 1999), 97-120; doi:10.1090/conm/241/03630.

CMR15 C. Ciliberto, M. Mendes Lopes, and X. Roulleau, On Schoen surfaces, Comment. Math. Helv. 90 (2015), no. 1, 59-74; doi:10.4171/CMH/346.

CPT03 C. Ciliberto, R. Pardini and F. Tovena, Regular canonical covers, Math. Nachr. 251 (2003), 19-27; doi:10.1002/mana.200310027.

Deb82 O. Debarre, Inégalités numériques pour les surfaces de type général (Appendice par A. Beauville), Bull. Soc. Math. France 110 (1982), no. 3, 319-346; doi:10.24033/ bsmf. 1965.

Dol96 I. V. Dolgachev, Mirror symmetry for lattice polarized K3 surfaces, J. Math. Sci. 81 (1996), no. 3, 2599-2630; doi:10.1007/BF02362332.

Dol12 Classical algebraic geometry. A modern view (Cambridge Univ. Press, Cambridge, 2012); doi:10.1017/CB09781139084437.

FH91 W. Fulton and J. Harris, Representation theory. A first course, Grad. Texts in Math., vol. 129 (Springer-Verlag, New York, 1991); doi:10.1007/978-1-4612-0979-9.

Gar17 A. Garbagnati, On K3 surface quotients of K3 or Abelian surfaces, Canad. J. Math. 69 (2017), no. 2, 338-372; doi:10.4153/CJM-2015-058-1.

GS16 A. Garbagnati and A. Sarti, Kummer surfaces and K3 surfaces with $(\mathbb{Z} / 2 \mathbb{Z})^{4}$ symplectic action, Rocky Mountain J. Math. 46 (2016), no. 4, 1141-1205; doi:10.1216/ RMJ-2016-46-4-1141.

Hun96 B. Hunt, The geometry of some special arithmetic quotients, Lecture Notes in Math., vol. 1637 (Springer-Verlag, Berlin, 1996); doi:10.1007/BFb0094399.

Liv81 R. A. Livne, On certain covers of the universal elliptic curve, Ph.D. Thesis, Harvard University, 1981.

MS63 Y. Matsushima and G. Shimura, On the cohomology groups attached to certain vector valued differential forms on the product of the upper half planes, Ann. of Math. 78 (1963), 417-449; doi:10.2307/1970534.

Nik75 V. V. Nikulin, Kummer surfaces, Math. USSR Izv. 9 (1975), no. 2, 261-275; doi:10.1070/ IM1975v009n02ABEH001477.

Nik76 _ Finite groups of automorphisms of Kählerian surfaces of type K3, Uspehi Mat. Nauk 31 (1976), no. 2, 223-224. 


\section{Rito, X. Roulleau and A. Sarti}

Nik80

Par91

Pia23

Pol06

PZ06

Rem07

Rit17

Sch07

Sai74

Shi63

Integral symmetric bilinear forms and some of their applications, Math. USSR Izv. 14 (1980), no. 1, 103-167; doi:10.1070/IM1980v014n01ABEH001060.

R. Pardini, Abelian covers of algebraic varieties, J. reine angew. Math. 417 (1991), 191-213; doi:10.1515/crll.1991.417.191.

M. Piazzolla-Beloch, Sulle superficie iperellittiche del $4^{\circ}$ ordine con 15 punti doppi, Rend. Circ. Mat. Palermo 47 (1923), 182-192; doi:10.1007/BF03014644.

F. Polizzi, Surfaces of general type with $p_{g}=q=1, K^{2}=8$ and bicanonical map of degree 2, Trans. Amer. Math. Soc. 358 (2006), no. 2, 759-798; doi:10.1090/S0002-994705-03673-1.

V. L. Popov and Yu. G. Zarhin, Finite linear groups, lattices, and products of elliptic curves, J. Algebra 305 (2006), no. 1, 562-576; doi:10.1016/j.jalgebra.2006.04.007.

L. Remy, Sur une famille dénombrable de surfaces hyperelliptiques du quatrième ordre, Bull. Soc. Math. France 35 (1907), 53-69; doi:10.24033/bsmf.792.

C. Rito, Explicit schoen surfaces: the Magma code, 2017, available at http://www.crito. utad.pt/schoen.pdf.

C. Schoen, A family of surfaces constructed from genus 2 curves, Internat. J. Math. 18 (2007), no. 5, 585-612; doi:10.1142/S0129167X07004175.

B. Saint-Donat, Projective models of $K-3$ surfaces, Amer. J. Math. 96 (1974), 602-639; doi: $10.2307 / 2373709$.

H. Shimizu, On discontinuous groups operating on the product of the upper half planes, Ann. of Math. 77 (1963), 33-71; doi:10.2307/1970201.

Carlos Rito crito@utad.pt

Universidade de Trás-os-Montes e Alto Douro, UTAD, Quinta de Prados, 5000-801 Vila Real, Portugal

Temporary address: Departamento de Matemática, Faculdade de Ciências da Universidade do Porto, Rua do Campo Alegre 687, 4169-007 Porto, Portugal

Xavier Roulleau Xavier.Roulleau@univ-amu.fr

Aix-Marseille Université, CNRS, Centrale Marseille, I2M UMR 7373, 13453 Marseille, France

Alessandra Sarti sarti@math.univ-poitiers.fr

Université de Poitiers, Laboratoire de Mathématiques et Applications, UMR 7348 du CNRS, Boulevard Pierre et Marie Curie, Téléport 2 - BP 30179, 86962 Futuroscope Chasseneuil, France 